\title{
A filtered-X LMS algorithm for sinusoidal reference signals - Effects of frequency mismatch
}

\author{
$\operatorname{AUTHOR}(\mathrm{S})$ :
}

Hinamoto, Y; Sakai, H

\section{CITATION:}

Hinamoto, Y ... [et al]. A filtered-X LMS algorithm for sinusoidal reference signals - Effects of frequency mismatch. IEEE SIGNAL PROCESSING LETTERS 2007, 14(4): 259-262

\section{ISSUE DATE:}

2007-04

URL:

http://hdl.handle.net/2433/50542

\section{RIGHT:}

(c)2007 IEEE. Personal use of this material is permitted. However, permission to reprint/republish this material for advertising or promotional purposes or for creating new collective works for resale or redistribution to servers or lists, or to reuse any copyrighted component of this work in other works must be obtained from the IEEE. 


\title{
A Filtered-X LMS Algorithm for Sinusoidal Reference Signals_Effects of Frequency Mismatch
}

\author{
Yoichi Hinamoto and Hideaki Sakai
}

\begin{abstract}
The objective of this letter is to analyze the effects of frequency mismatch for an adaptive algorithm that becomes the Filtered-X LMS algorithm when the reference signals are purely sinusoidal. The Filtered-X LMS algorithm is often used for active control of acoustic noise. For the case of sinusoidal noise sources, if there is a deviation between the frequency used in the adaptive algorithm and its true value (frequency mismatch), the performance of the Filtered-X LMS algorithm might degrade considerably. In this letter, using the equivalent transfer function method, the effects of frequency mismatch are analyzed precisely. Finally, computer simulations are presented to demonstrate the obtained results.
\end{abstract}

Index Terms-Active noise control (ANC), Filtered-X LMS algorithm, frequency mismatch.

\section{INTRODUCTION}

A CTIVE noise control (ANC) is well known as a useful technique for suppressing acoustic noise [1], [2]. The ANC system produces the antinoise field to cancel the primary sound field from the noise sources by destructive interference of sound fields. The primary noise sources are often generated from rotating machines, and in such cases, acoustic noise can be treated as periodic signals that can be represented by a superposition of sinusoidal signals. We can find typical applications in the propeller aircraft, motorboats, helicopters, etc. [3], [4].

In the adaptive algorithms for ANC, a frequency analyzer, e.g., tachometer, provides the signal frequencies, which are assumed to be the true ones, in advance. However, if there is a deviation between the frequency used in the adaptive algorithm and its true value (frequency mismatch), the performance of the adaptive algorithm in the ANC system might degrade considerably. In [5] and [6], the effects of frequency mismatch have been analyzed using the averaging technique for the case where the reference signals are real sinusoids and the secondary path is not considered. Though the technique is based on the assumption that the step size is very small, the obtained results are in good agreement with simulated values for relatively large step size.

When the effects of the secondary path cannot be ignored, we need to use the Filtered-X LMS (FXLMS) algorithm. The objective of this letter is to analyze the effects of frequency mismatch accurately on the FXLMS algorithm with sinusoidal reference

Manuscript received May 29, 2005; revised August 15, 2005. The associate editor coordinating the review of this manuscript and approving it for publication was Dr. Philip Schniter.

The authors are with the Department of Systems Science, Graduate School of Informatics, Kyoto University, Kyoto 606-8501, Japan (e-mail: hinamoto@sys.i.kyoto-u.ac.jp; hsakai@i.kyoto-u.ac.jp).

Digital Object Identifier 10.1109/LSP.2006.884901

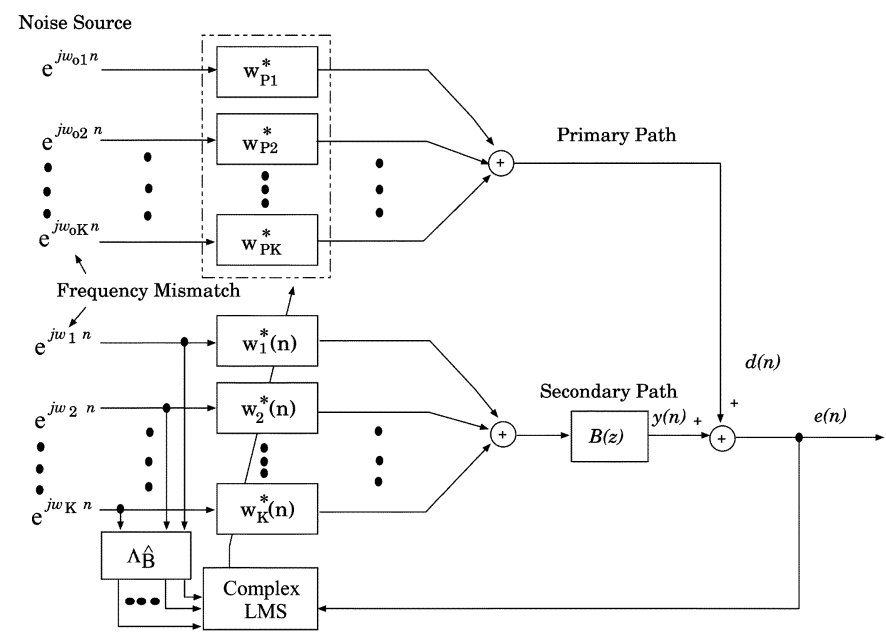

Fig. 1. Block diagram of the whole ANC system with sinusoidal reference signals.

signals. Using the equivalent transfer function method used in [7] and [8], the exact steady-state error signal and trajectory of the tap weight vector due to the frequency mismatch are derived. Finally, computer simulations are presented to demonstrate the validity of the obtained results.

\section{Active Control of Sinusoidal Reference SIGNALS BY THE FXLMS ALGORITHM}

In this section, we consider the case with no frequency mismatch. The block diagram of the ANC system is shown in Fig. 1. The noise source of the $i$ th rotating machine is assumed to be narrow band and is modeled by a complex sinusoidal wave [3]. Namely, the $i$ th reference signal that is an input to the adaptive system is given by $x_{i}(n)=e^{j \omega_{i} n}(i=1,2, \ldots, K)$, where $K$ denotes the total number of noise sources, and $\omega_{i}=2 \pi f_{i} / f_{s}$ with the frequency $f_{i}$ of the $i$ th rotating machine and the sampling frequency $f_{s}$. When the higher harmonics besides the fundamental frequency are generated, each harmonic sinusoidal signal is treated as the input to the adaptive system. Let the reference signal vector $\boldsymbol{x}(n)$ and the tap weighting vector $\boldsymbol{w}(n)$ be defined by

$$
\begin{aligned}
\boldsymbol{x}(n) & =\left[\begin{array}{llll}
e^{j \omega_{1} n} & e^{j \omega_{2} n} & \ldots & e^{j \omega_{K} n}
\end{array}\right]^{T} \\
\boldsymbol{w}(n) & =\left[\begin{array}{llll}
w_{1}(n) & w_{2}(n) & \ldots & w_{K}(n)
\end{array}\right]^{T}
\end{aligned}
$$

where $(\cdot)^{T}$ stands for the transposition, the frequency $\omega_{i}$ is known, and $\omega_{i} \neq \omega_{j}$ for $i \neq j$. The transfer function of the secondary path from the loudspeaker to the error microphone is assumed to be an $L$ th-order finite impulse response (FIR) system $B(z)=b_{0}+b_{1} z^{-1}+\cdots+b_{L} z^{-L}$. The output of the 
adaptive system passes through the secondary path to form the antinoise signal $y(n)$, which can be expressed as

$$
y(n)=\sum_{l=0}^{L} b_{l} \sum_{i=1}^{K} w_{i}^{*}(n-l) x_{i}(n-l)
$$

where $(\cdot)^{*}$ denotes the complex conjugate. Each sinusoidal reference signal $x_{i}(n)=e^{j \omega_{i} n}$ passes through each primary path with the transfer function $P_{i}(z)(i=1,2, \ldots, K)$ to yield the desired signal $d(n)$ at the error microphone, which is written as

$$
d(n)=\sum_{i=1}^{K} P_{i}\left(e^{j \omega_{i}}\right) e^{j \omega_{i} n} \equiv \sum_{i=1}^{K} w_{P i}^{*} e^{j \omega_{i} n}=\boldsymbol{w}_{P}^{H} \boldsymbol{x}(n)
$$

in the steady state, where $(\cdot)^{H}$ denotes the Hermitian transpose, and for notational simplicity, we define $w_{P i}^{*}=P_{i}\left(e^{j \omega_{i}}\right)$ and

$$
\boldsymbol{w}_{P}=\left[\begin{array}{llll}
w_{P 1} & w_{P 2} & \ldots & w_{P K}
\end{array}\right]^{T} .
$$

The error signal is given by

$$
e(n)=d(n)+y(n) .
$$

Using the estimated transfer function $\hat{B}(z)$ of $B(z)$, the filtered reference vector by $\hat{B}(z)$ is written as $\Lambda_{\hat{B}} x(n)$, where

$$
\begin{aligned}
\Lambda_{\hat{B}} & =\operatorname{diag}\left[\hat{B}_{1}, \hat{B}_{2}, \ldots, \hat{B}_{K}\right] \\
\hat{B}_{i} & =\sum_{l=0}^{M} \hat{b}_{l} e^{-j \omega_{i} l} \quad(i=1,2, \ldots, K)
\end{aligned}
$$

and $M$ may be different from $L$. So, the FXLMS algorithm in this case is described by

$$
\boldsymbol{w}(n+1)=\boldsymbol{w}(n)-\mu \Lambda_{\hat{B}} \boldsymbol{x}(n) e^{*}(n)
$$

where $\mu$ is a positive step size. This is a special case of the multireference frequency FXLMS algorithm in [1, pp.131-134] with a single weight for each frequency. Equations (3)-(6) describe the dynamics of the ANC system. The analysis of this system has been done in [9] using the state-space technique in linear system theory where the stability limit about $\mu$ has been shown.

\section{EFFECTS OF FREQUENCY MisMatch}

In some situations, the frequencies in the desired signal (4) are not precisely known. In this section, using the equivalent transfer function technique, we analyze the effects of frequency mismatch for the FXLMS algorithm with sinusoidal reference signals. In this case, the frequencies in the FXLMS algorithm (6) differ from those in the desired signal

$$
d(n)=\sum_{i=1}^{K} w_{P i}^{*} e^{j \omega_{o i} n} .
$$

The quantity $\Delta \omega_{i}=\omega_{o i}-\omega_{i}(i=1,2, \ldots, K)$ denotes the frequency mismatch. The complex conjugate of the $k$ th component of (6) is written as

$$
w_{k}^{*}(n+1)=w_{k}^{*}(n)-\mu \hat{B}_{k}^{*} e^{-j \omega_{k} n} e(n) .
$$

Taking the $z$-transform of (8), we have

$$
z W_{k}(z)=W_{k}(z)-\mu \hat{B}_{k}^{*} E\left(z e^{j \omega_{k}}\right)
$$

where

$$
W_{k}(z)=\sum_{n=0}^{\infty} w_{k}^{*}(n) z^{-n}, \quad E(z)=\sum_{n=0}^{\infty} e(n) z^{-n} .
$$

Then, the $z$-transform of the output of the adaptive system $\sum_{i=1}^{K} w_{i}^{*}(n) e^{j \omega_{i} n}$ is $\sum_{i=1}^{K} W_{i}\left(z e^{-j \omega_{i}}\right)$. From (3) and (5), we have

$$
E(z)=D(z)+B(z) \sum_{k=1}^{K} W_{k}\left(z e^{-j \omega_{k}}\right)
$$

but from (9), we have

$$
W_{k}(z)=-\frac{\mu \hat{B}_{k}^{*}}{z-1} E\left(z e^{j \omega_{k}}\right) .
$$

Hence, from (11) and (12)

$$
E(z)=D(z)-B(z) A(z) E(z)
$$

where

$$
A(z)=\mu \sum_{k=1}^{K} \frac{\hat{B}_{k}^{*}}{z e^{-j \omega_{k}}-1}
$$

and $D(z)$ is the $z$-transform of $d(n)$ in (7).

From (13), it follows that

$$
E(z)=\frac{1}{1+B(z) A(z)} D(z) .
$$

Thus, the transfer function from $d(n)$ to $e(n)$ is given by $(1+$ $B(z) A(z))^{-1}$. The characteristic equation $1+B(z) A(z)=$ 0 is the same as that derived in [9] where the complex conjugate version is presented. In [9], this result is obtained by the state-space technique in linear system theory that requires vector-matrix manipulations, whereas only scalar operations are used in (8)-(14). Assuming that $\mu$ is taken within the stability region derived in [9], based on the definition of frequency response for (7) $e(n)$ in the steady state is given by

$$
e(n)=\sum_{i=1}^{K} \frac{1}{1+\gamma_{i}^{*}} w_{P i}^{*} e^{j \omega_{o i} n}
$$

where from (14)

$$
\gamma_{i}=B^{*}\left(e^{j \omega_{o i}}\right) A^{*}\left(e^{j \omega_{o i}}\right)=\mu B_{o i}^{*} \sum_{k=1}^{K} \frac{\hat{B}_{k}}{e^{-j\left(\omega_{o i}-\omega_{k}\right)}-1} .
$$

Substitution of (15) into (12) gives

$$
W_{k}(z)=-\frac{\mu \hat{B}_{k}^{*}}{z-1} \frac{1}{1+B\left(z e^{j \omega_{k}}\right) A\left(z e^{j \omega_{k}}\right)} D\left(z e^{j \omega_{k}}\right) .
$$


Noting that $D\left(z e^{j \omega_{k}}\right)$ corresponds to $d(n)$ in (7) with $w_{o i}$ replaced by $\omega_{o i}-\omega_{k}$, the stationary trajectory $w_{o k}(n)$ of $w_{k}(n)$ is given by

$$
\begin{aligned}
w_{o k}^{*}(n)=-\mu \hat{B}_{k}^{*} \sum_{i=1}^{K} & w_{P i}^{*} \frac{e^{j\left(\omega_{o i}-\omega_{k}\right) n}}{\left(e^{j\left(\omega_{o i}-\omega_{k}\right)}-1\right)} \\
& \cdot \frac{1}{\left(1+\left.B\left(z e^{j \omega_{k}}\right) A\left(z e^{j \omega_{k}}\right)\right|_{\left.z=e^{j\left(\omega_{o i}-\omega_{k}\right)}\right)}\right.} .
\end{aligned}
$$

Hence, we have

$$
w_{o k}(n)=-\mu \hat{B}_{k} \sum_{i=1}^{K} \frac{w_{P i} e^{-j\left(\omega_{o i}-\omega_{k}\right) n}}{\left(e^{-j\left(\omega_{o i}-\omega_{k}\right)}-1\right)\left(1+\gamma_{i}\right)}
$$

When $\Delta \omega_{k}=\omega_{o k}-\omega_{k}(k=1,2, \ldots, K)$ are sufficiently small, for $\gamma_{k}$, the $k$ th term in (17) is dominant, and in (18), so is the $k$ th term. Hence, the stationary trajectory $w_{o k}(n)$ of the tap weight $w_{k}(n)$ is approximated as

$$
w_{o k}(n) \simeq-e^{-j \Delta \omega_{k}(n-1)} \frac{\mu \hat{B}_{k} w_{P k}}{1-e^{j \Delta \omega_{k}}+\mu B_{o k}^{*} \hat{B}_{k} e^{j \Delta \omega_{k}}} .
$$

Moreover, under the condition $\left|\Delta \omega_{k}\right| \ll\left|B_{o k}^{*} \hat{B}_{k} \mu\right|$, it fol-

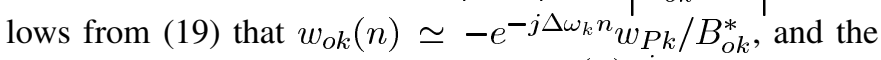
output of the $k$ th adaptive weight $w_{o k}^{*}(n) e^{j \omega_{k} n}$ is close to $-e^{j \omega_{o k} n} w_{P k}^{*} / B_{o k}$, which cancels the corresponding component in $d(n)$, and the error signal $e(n)$ approaches zero. Otherwise, the error signal does not approach zero, and the effect of frequency mismatch appears in the error signal. It is noted that in the case of no frequency mismatch, that is, $\omega_{o i}=\omega_{i}$, it follows that $\gamma_{i}=\infty$, and (16) is zero as it should be. The increase in the time-averaged squared magnitude of the error signal due to the frequency mismatch is given by

$$
\operatorname{MISMATCH}=\sum_{i=1}^{K} \frac{1}{\left|\gamma_{i}+1\right|^{2}}\left|w_{P i}\right|^{2}
$$

In the case when the frequency mismatch $\Delta \omega_{i}$ is sufficiently small, we can approximate (20) as

$$
\begin{aligned}
\operatorname{MISMATCH} & \simeq \sum_{i=1}^{K} \frac{2\left(1-\cos \Delta \omega_{i}\right)}{\mu^{2}\left|B_{o i}\right|^{2}\left|\hat{B}_{i}\right|^{2}}\left|w_{P i}\right|^{2} \\
& \simeq \sum_{i=1}^{K} \frac{\left(\Delta \omega_{i}\right)^{2}}{\mu^{2}\left|B_{o i}\right|^{2}\left|\hat{B}_{i}\right|^{2}}\left|w_{P i}\right|^{2}
\end{aligned}
$$

To compare (20) and (21) with the existing result in [5], we consider a special case of single real sinusoid without the secondary path $(B(z)=\hat{B}(z)=1)$. Writing $w_{P 1}=a / 2+j b / 2$ and $w_{P 2}=a / 2-j b / 2$, then $d(n)=w_{P 1}^{*} e^{j \omega n}+w_{P 2}^{*} e^{-j \omega n}=$ $a \cos \omega n+b \sin \omega n$, where $A=\sqrt{a^{2}+b^{2}}=2\left|w_{P 1}\right|$. If the adaptive tap weights $w_{1}(n)$ and $w_{2}(n)$ are of complex conjugate relation, so are $w_{1}(n+1)$ and $w_{2}(n+1)$. This is seen as follows. Writing $w_{1}(n)=a(n) / 2+j b(n) / 2$, and $w_{2}(n)=a(n) / 2-j b(n) / 2$, we have $y(n)=$ $w_{1}^{*}(n) e^{j \omega n}+w_{2}^{*}(n) e^{-j \omega n}=a(n) \cos \omega n+b(n) \sin \omega n$. Hence, $e(n)$ is real, and (6) is written as

$$
\begin{aligned}
& w_{1}(n+1)=w_{1}(n)-\mu e^{j \omega n} e(n) \\
& w_{2}(n+1)=w_{2}(n)-\mu e^{-j \omega n} e(n) .
\end{aligned}
$$

Thus, $w_{1}(n+1)=w_{2}^{*}(n+1)$. Hence

$$
\begin{aligned}
& a(n+1)=a(n)-2 \mu e(n) \cos \omega n \\
& b(n+1)=b(n)-2 \mu e(n) \sin \omega n
\end{aligned}
$$

This is used in [5], and the effect of frequency mismatch $\Delta \omega=$ $\omega_{o}-\omega$ is analyzed by the averaging method, and the corresponding formula to (20) in [5] is

$$
\frac{A^{2}}{2} \frac{4\left(\cos \omega_{o}-\cos \omega\right)^{2}}{(2-2 \mu)^{2}\left(\cos \omega_{o}-\cos \omega\right)+4 \mu^{2} \sin ^{2} \omega_{o}}
$$

where in (23), $\mu$ in [5] is replaced by $2 \mu$ due to (22). For $|\Delta \omega| \ll$ $1,(23)$ is approximated as

$$
\frac{A^{2}}{2} \frac{4}{(2-2 \mu)^{2}+\frac{4 \mu^{2}}{(\Delta \omega)^{2}}}
$$

but (21) is $A^{2}(\Delta \omega)^{2} / 2 \mu^{2}$ so that (24) coincides with this for the case where $\mu^{2} /(\Delta \omega)^{2} \gg 1$.

For the general real-valued system with multiple sinusoids and secondary path, we set a pair of frequency $\omega_{i}$ and $-\omega_{i}$ with the complex conjugate pair of initial tap weights.

\section{Simulation Results}

To examine the validity of the above theoretical developments, some simulations have been performed. An FIR system with 256 taps has been used as the transfer function $B(z)$. This corresponds to the transfer function of a real secondary path in [1]. The above FIR system has been truncated with 32 taps and used as an estimated transfer function $\hat{B}(z)$ instead of $B(z)$. It is seen that $B(z)$ and $\hat{B}(z)$ satisfy the necessary condition (the $90^{\circ}$ condition) for convergence in [9] as follows:

$$
\operatorname{Re}\left\{B_{i}^{*} \hat{B}_{i}\right\}>0 \quad(i=1,2, \ldots, K) .
$$

The reference signals are assumed to be of the form of unit magnitude complex sinusoid. In our simulation studies, we consider three tonal noise frequencies of 125 , 250 , and $300 \mathrm{~Hz}$, where $250 \mathrm{~Hz}$ is assumed to be the harmonic of $125 \mathrm{~Hz}$. If a sampling frequency is $1 \mathrm{kHz}$, then $\omega_{1}=(\pi / 4), \omega_{2}=(\pi / 2)$, and $\omega_{3}=(3 \pi / 5)$. In this case, the reference signal vector is given by $\boldsymbol{x}(n)=$

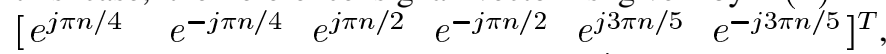
and $K=6$. Each primary noise source $e^{j \omega_{o i} n}$ passes through either of two primary paths $P_{1}(z)$ and $P_{2}(z)$ in [1]. Hence, the frequency responses from the primary noise sources to the microphone are given by $\boldsymbol{w}_{P}=\left[P_{1}\left(e^{j \omega_{o 1}}\right), P_{1}\left(e^{-j \omega_{01}}\right)\right.$, $\left.P_{1}\left(e^{j \omega_{o 2}}\right), P_{1}\left(e^{-j \omega_{o 2}}\right), P_{2}\left(e^{j \omega_{o 3}}\right), P_{2}\left(e^{-j \omega_{o 3}}\right)\right]^{H}$. It turns out that in this case, $\mu_{\max }=0.5167$ corresponds to the stability 


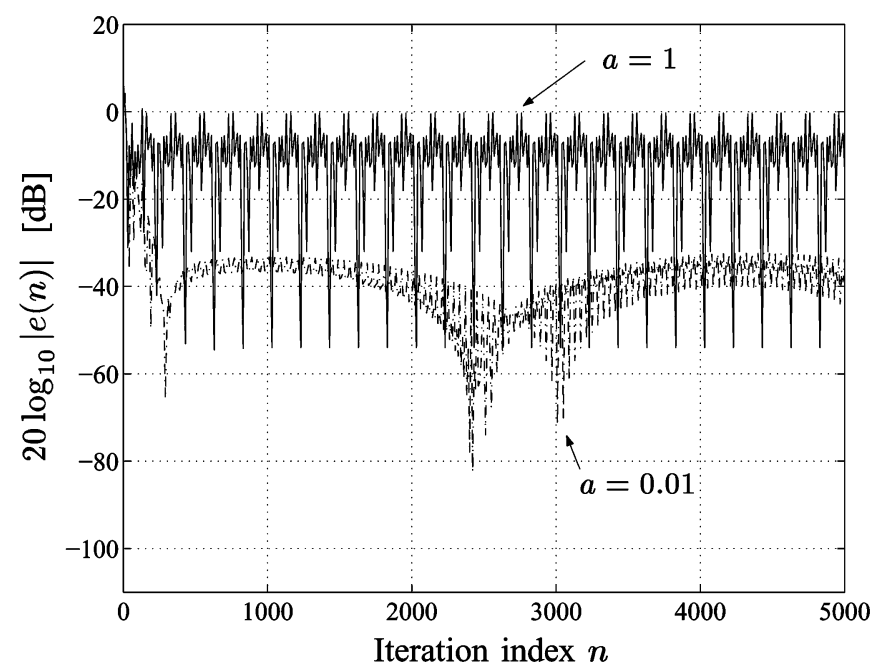

Fig. 2. Learning curves in decibels when the frequency mismatch exists. (True signal frequencies: $\omega_{o 1}=\pi / 4+a \times 10^{-2} \pi, \omega_{o 2}=\pi / 2+3 a \times 10^{-2} \pi$, $\omega_{o 3}=3 \pi / 5+a \times 10^{-2} \pi$. User-specified frequencies: $\omega_{1}=\pi / 4, \omega_{2}=$ $\left.\pi / 2, \omega_{3}=3 \pi / 5\right)$.

TABLE I

EXPERIMENTAL AND THEORETICAL VALUES OF THE INCREASE IN THE TIME-AvERAGED SQUARED MAGNITUdE OF THE ERROR Signals Due to the FREQUENCY Mismatch With the SAME EXPERIMENTAL CONDITIONS IN FIG. 2.

\begin{tabular}{lcc}
\hline & $a=1$ & $a=0.01$ \\
\hline experimental value [dB] & -6.92 & -37.70 \\
value in (20) [dB] & -6.92 & -37.70 \\
value in (21) [dB] & -2.52 & -37.66 \\
\hline
\end{tabular}

limit of the step size [9]. So, we adopted $\mu=0.3$. The initial weights are set to $w_{i}(0)=1(i=1,2, \ldots, K)$.

Fig. 2 shows the learning curves in the presence of the frequency mismatch. In conjunction with this figure, in Table I, we compare the experimental and theoretical values in (20) and (21) of the increase in the time-averaged squared magnitude of the error signal due to the frequency mismatch. From Table I, since
(20) gives an exact solution, the result perfectly coincides with the experimental one. On the other hand, (21) is derived under the assumption that the frequency mismatch $\Delta \omega_{i}$ is sufficiently small. So if the frequency mismatch is large as in the case $a=1$ (compared with the case of $a=0.01$ ), the difference between the theoretical and experimental values is relatively large.

\section{CONCLUSION}

This letter has investigated the exact effects of the frequency mismatch for the FXLMS algorithm with sinusoidal reference signals using the equivalent transfer function technique. The exact steady-state expression for tap weights and the error signal have been derived. The simulation results have demonstrated the validity of the derived expressions.

\section{REFERENCES}

[1] S. M. Kuo and D. R. Morgan, Active Noise Control Systems. New York: Wiley, 1996

[2] C. R. Fuller, S. J. Elliot, and P. A. Nelson, Active Control of Vibrations. New York: Academic, 1996.

[3] S. Johansson, S. Nordebo, and I. Claesson, "Convergence analysis of a twin-reference complex least-mean-squares algorithm," IEEE Trans. Speech Audio Process., vol. 10, no. 3, pp. 213-221, May 2002.

[4] S. Johansson, I. Claesson, S. Nordebo, and P. Sjösten, "Evaluation of multiple reference active noise control algorithms on Dornier 328 aircraft data," IEEE Trans. Speech Audio Process., vol. 7, no. 4, pp. 473-477, Jul. 1999.

[5] Y. Xiao and Y. Tadokoro, "LMS-based notch filter for the estimation of sinusoidal signals in noise," Signal Process., vol. 46, pp. 223-231, 1995.

[6] Y. Xiao, A. Ikuta, L. Ma, L. Xu, and R. K. Ward, "Statistical properties of the LMS Fourier analyzer in the presence of frequency mismatch," IEEE Trans. Circuits Syst. I, vol. 51, no. 12, pp. 2504-2515, Dec. 2004.

[7] J. R. Glover, Jr, "Adaptive noise canceling applied to sinusoidal interferences," IEEE Trans. Acoust., Speech, Signal Process., vol. ASSP-25, no. 6, pp. 484-491, Dec. 1977.

[8] S. J. Elliott, I. M. Stothers, and P. A. Nelson, "A multiple error LMS algorithm and its application to active control of sound and vibration," IEEE Trans. Acoust., Speech, Signal Process., vol. ASSP-35, no. 5, pp. 1423-1434, Oct. 1987.

[9] Y. Hinamoto and H. Sakai, "Analysis of the filtered-X LMS algorithm and a related new algorithm for active control of multitonal noise," IEEE Trans. Speech Audio Process., vol. 14, no. 1, pp. 123-130, Jan. 2006. 